\title{
An observational study to compare between conventional condom balloon catheter with CG balloon
}

\author{
Somila Xess ${ }^{1 *}$, Preeti Baghel ${ }^{2}$ \\ ${ }^{1}$ Department of Obstetrics and Gynecology, GMC, Ambikapur, Chhattisgarh, India \\ ${ }^{2}$ Department of Obstetrics and Gynecology, SMS Medical College, Jaipur, Rajasthan, India
}

Received: 02 December 2018

Accepted: 07 January 2019

\section{*Correspondence:}

Dr. Somila Xess,

E-mail: somilaxess05@gmail.com

Copyright: (c) the author(s), publisher and licensee Medip Academy. This is an open-access article distributed under the terms of the Creative Commons Attribution Non-Commercial License, which permits unrestricted non-commercial use, distribution, and reproduction in any medium, provided the original work is properly cited.

\begin{abstract}
Background: Postpartum haemorrhage (PPH) is a life-threatening complication of delivery. The most common cause of PPH is uterine atony. Intrauterine balloon tamponade has been suggested as an effective, easily administered minimally invasive treatment option to control uterine bleeding while preserving the mother's ability to bear additional children.

Methods: Twenty women with normal vaginal delivery were studied over a period of six months, 10 were inserted conventional balloon tamponade and rest 10 were inserted CG balloon and outcome studied in terms of time to assemble, leakage, expulsion, lumen occlusion, volume of fluid used, time to arrest bleeding, cost, drainage port, inflation deflation interval.

Results: Most of the women were para 4 or more, unbooked belonging to age group of 20-30. Mean time to assemble Condom balloon tamponade was $1.8 \mathrm{~min}$ and that in CG balloon was 1.2 minute. There was leakage and expulsion in two and lumen occlusion in three in the conventional balloon catheter. In the second group there was no leakage ,lumen occlusion or expulsion. There is uterine drainage port present in CG balloon which is characteristic of it which helps in determining the actual blood loss in real time. The inflation deflation interval and mean volume of fluid are almost same in both the groups.

Conclusions: Both conventional balloon and CG balloon are effective and lifesaving in low resource setting with few advantages of $\mathrm{CG}$ balloon over conventional balloon tamponade.
\end{abstract}

Keywords: CG balloon, Conventional balloon tamponade, PPH

\section{INTRODUCTION}

The most common cause of PPH is uterine atony; up to $80 \%$ of the cases result from suboptimal contraction of the myometrium following placental separation. The repertoires of the management measures can be generally divided into operative and nonoperative interventions. In acute postpartum haemorrhage refractory to medical and other conservative interventions, invasive therapies may include arterial embolization, uterine compression sutures, uterine artery ligation, and, ultimately, hysterectomy. However, these measures are highly invasive, require extensive resources, expertise, and are associated with significant morbidities.

Intrauterine balloon tamponade has been suggested as an effective, easily administered minimally invasive treatment option to control uterine bleeding while preserving the mother's ability to bear additional children. Multiple types of balloons are available, including Bakri balloon, BT-Cath balloon tamponade catheter, Foley catheters, Rusch balloon, condom 
catheters and the Sengstaken-Blakemore tube. In term of mechanism of action, the intrauterine balloon is believed to act by exerting inward to outward pressure against the uterine wall, resulting in a reduction in persistent capillary and venous bleeding from the endometrium and the myometrium.

As per WHO recommendations, the use of intrauterine balloon tamponade (UBT) is recommended for the treatment of PPH due to uterine atony if women do not respond to uterotonics. ${ }^{1}$ FIGO also included uterine balloon tamponade as a recommended second-line intervention for the treatment of $\mathrm{PPH}^{2}$ The successful outcome of balloon tamponade is reported to be 80-100 $\% .^{3}$ This high efficacy avoids surgery which is often delayed or may be unavailable thereby costing the women her life. Intrauterine balloon may also be used as a temporizing measure while awaiting transfer or to resuscitate her.

The aim and objectives of the experiment are: to establish the efficacy of balloon tamponade, to compare between conventional condom balloon catheter with CG balloon.

\section{METHODS}

This prospective study was carried out in GMC Ambikapur over a period of 6 months from June 2018 to November 2018.Total of 20 patients delivered vaginally were included and inclusion and exclusion criteria established. Ten women were inserted conventional condom balloon catheter and rest 10 were inserted CG balloon and statistical analysis done.

\section{Inclusion criteria}

- Women with vaginal delivery

- Medical measures of PPH tried but failed.

- Atonic PPH

- Active Management of $3^{\text {rd }}$ stage done.

\section{Exclusion criteria}

- Traumatic PPH

- Shock more than 2 hours

- Retained Placenta

- Known uterine anomaly

- DIC.

\section{Conventional balloon catheter}

With aseptic precautions, a condom was rolled over proximal one third of a Foley catheter (No. 20) and tied with a cotton thread or vicryl/silk on two sites $1 \mathrm{~cm}$ apart. The cervix was identified, and the condom tamponade was introduced into the uterus manually. The condom was inflated with $100-500 \mathrm{ml}$ warm saline with the help of a $50 \mathrm{ml}$ syringe or iv set till bleeding ceased. The catheter was tightened by an umbilical clamp or cotton string and taped to the thigh. Vagina was loosely packed.

\section{CG balloon}

It was named 'CG Balloon', as it was invented in Chhattisgarh India. ${ }^{4}$ It is prepared manually with all aseptic precautions as follows.

Collect a Foley's catheter of size 20-22, a packed condom, scissors, two $20 \mathrm{ml}$ Syringes and $500 \mathrm{ml}$ bottle of saline in a tray. From the drainage tube of the catheter, cut two rings of approximately 1-2 mm width (Figure 1a). Excise (not merely incise) the bulb of the catheter after inflating it with air (Figure 1b). Unfold the condom over distal one-third of the catheter (Figure 1c). Use these Rings encircling twice only (like a rubber band in a ponytail) to secure the condom over catheter leaving 1.5$2 \mathrm{~cm}$ from both the ends of condom (Figure 1d). Excise the tip of the Foley's catheter and condom together to facilitate drainage of blood i.e. uterine drainage port (Figure 1e). Wash the device with antiseptic solution. In both the groups insertion was done after holding cervical lips with sponge holding forceps and with two fingers gently introduced so that the lower tied end remains just above internal os.

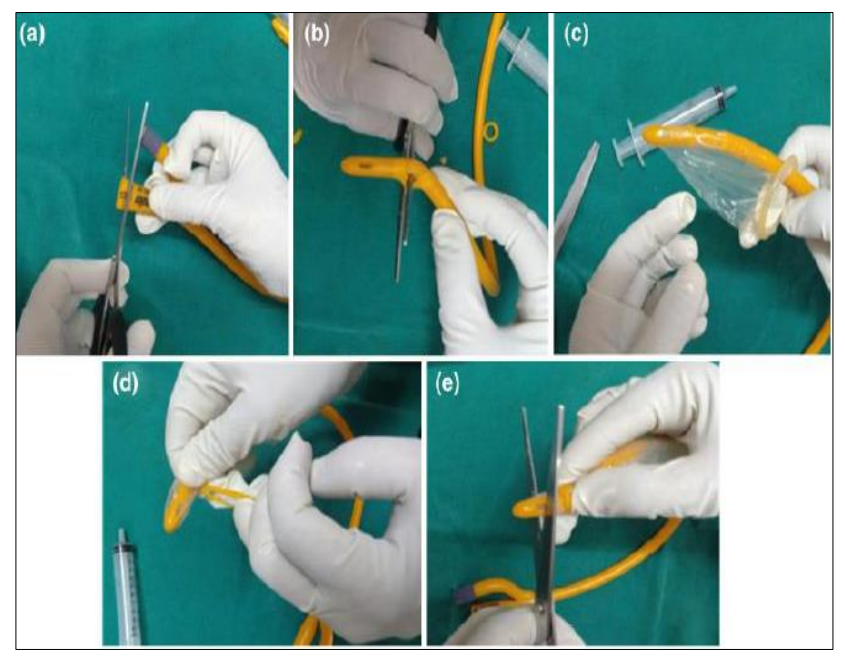

Figure 1: Assembly of CG balloon.

Both the groups were compared on the basis of time to assemble, time to achieve haemostasis, leakage, lumen occlusion, cost, expulsion, drainage port, inflation deflation interval, volume of fluid used.

\section{RESULTS}

Table 1 shows sociodemographic profile of patients, total of 20 women were studied over a period of six months. $50 \%$ were para 4 or more, those with parity 3 and 2 were $25 \%$ and $15 \%$ respectively whereas primipara were $10 \%$.

Most of the patients (40\%) belonged to the age group of 20-30 years and 10\% were more than 40 years of age. $75 \%$ were unbooked and referred from periphery and $25 \%$ were booked showing the importance of ANC visits and early management of PPH. 
Table 1: Sociodemographic profile of patients.

\begin{tabular}{|l|l|}
\hline Parity & Number of women (n) \\
\hline 1 & $2(10 \%)$ \\
\hline 2 & $3(15 \%)$ \\
\hline 3 & $5(25 \%)$ \\
\hline $\overrightarrow{4}$ & $10(50 \%)$ \\
\hline Age & Number of women $(\mathbf{n})$ \\
\hline$<20$ & $4(20 \%)$ \\
\hline $20-30$ & $8(40 \%)$ \\
\hline $30-40$ & $6(30 \%)$ \\
\hline$>40$ & $2(10 \%)$ \\
\hline ANC visits & Number of women $(\mathbf{n})$ \\
\hline Booked & $5(25 \%)$ \\
\hline Unbooked & $15(75 \%)$ \\
\hline
\end{tabular}

Table 2 compares the characteristics of two balloon catheter. It was observed that the mean time to assemble Condom balloon tamponade was 1.8 minutes and that in CG balloon was 1.2 minutes.

Table 2: Comparison of two balloon catheter.

\begin{tabular}{|l|l|l|}
\hline Criteria & $\begin{array}{l}\text { Conventional } \\
\text { balloon }\end{array}$ & CG balloon \\
\hline Time to assemble & $1.8 \mathrm{~min}$ (mean) & 1.2 min (mean) \\
\hline Leakage & $2(20 \%)$ & 0 \\
\hline Lumen occlusion & $3(30 \%)$ & 0 \\
\hline Drainage port & Absent & Present \\
\hline Mean volume of fluid & $300 \mathrm{ml}$ & $250 \mathrm{ml}$ \\
\hline $\begin{array}{l}\text { Time to arrest } \\
\text { bleeding }\end{array}$ & $6.6 \mathrm{~min}$ (mean) & $6 \mathrm{~min}$ (mean) \\
\hline Expulsion & $2(20 \%)$ & 0 \\
\hline $\begin{array}{l}\text { Inflation deflation } \\
\text { interval }\end{array}$ & 14.4 hour & 12 hour \\
\hline Cost & Costly & Cheap \\
\hline
\end{tabular}

There was leakage and expulsion in two and lumen occlusion in three in the conventional balloon catheter. In the second group there was no leakage, lumen occlusion or expulsion.

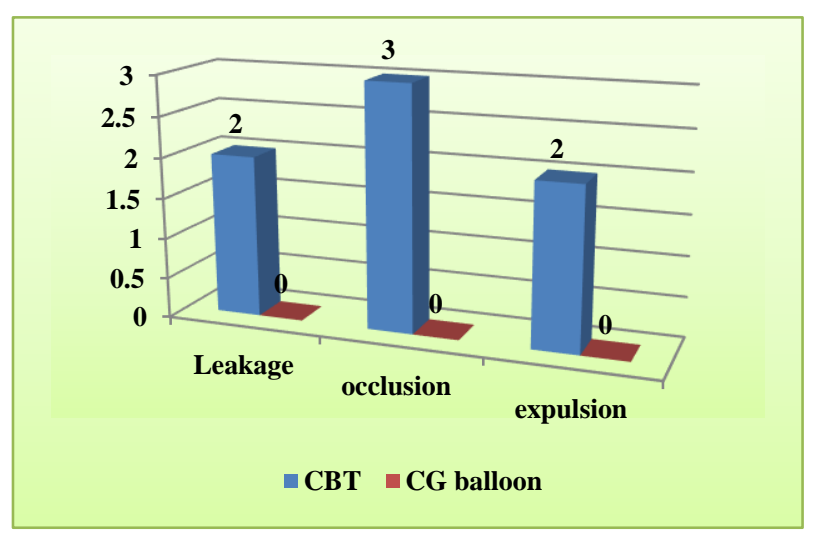

Figure 2: Comparison of two groups.

There is uterine drainage port present in CG balloon which is characteristic of it which helps in determining the actual blood loss in real time. The inflation deflation interval and mean volume of fluid are almost same in both the groups.

Figure 2 depicts there was no leakage, occlusion or expulsion in CG balloon whereas there were 2 cases of leakage and expulsion whereas occlusion was 3.

\section{DISCUSSION}

UBT (Uterine balloon tamponade) treats PPH due to uterine atony when uterotonics and bimanual compression fail to stop bleeding. It reduces the need for surgical intervention and blood transfusion and can be used during transfer. UBT should be part of the PPH emergency kit. In present study, we have compared conventional balloon catheter with that of CG balloon in 20 subjects. It was observed that the mean time to assemble Condom balloon tamponade was $1.8 \mathrm{~min}$ and that in CG balloon was 1.2 minute. This was similar to the study done by Dalia Y et al. ${ }^{5}$ The mean time were taken to assemble the apparatus was $1 \mathrm{~min} 26 \mathrm{sec}$ and the fastest to assemble was CBT with tip cut and CG Balloon. There was leakage and expulsion in two and lumen occlusion in three in the conventional balloon catheter. In the second group there was no leakage, lumen occlusion or expulsion. Similar results were obtained in the study done by Dalia Y et al, in which the leakage, expulsion and lumen occlusion were higher with conventional balloon tamponade than with CG balloon and tip cut condom. ${ }^{5}$ These varying results could be due to use of thread for tying the ends which could remain loose to create a leakage or expulsion or tight which could be the reason for lumen occlusion. Also the knot tying requires some level of expertise which is not seen with CG balloon in which bands of catheter are used for the ends. The mean volume of fluid used to create tamponade was $300 \mathrm{ml}$ in Conventional balloon tamponade and in CG balloon it was $250 \mathrm{ml}$. In the study done by Mishra $\mathrm{N}$ et al the mean volume of fluid used was $245.7 \mathrm{ml}^{4}$

One of the best things about CG balloon is the presence of drainage port through which authors can measure bleeding in real time which makes it better than conventional balloon tamponade. In present study the mean time to arrest bleeding was 6.6 minute in conventional balloon tamponade and 6 minute in CG balloon and hence not so much difference is noted which was comparable to the study by Rathore AM et al,(6.06 minutes). ${ }^{6}$ Mishra $\mathrm{N}$ et al noted mean time to arrest bleeding as 12.69 minutes which is almost double as that of present study which could be due to associated conditions apart from atonicity. ${ }^{4}$ The inflation deflation interval was almost similar in both the groups with mean of 14.4 hour and 12 hours respectively in CBT and CG balloon which is again similar to the study done by Mishra $\mathrm{N}$ et al. ${ }^{4}$ In present study $60 \%$ had tamponade for 14 hours. Y. Dalia et al noted mean duration of $22.56 \pm 3.84$ hours which is slightly higher than ours. ${ }^{5}$ 
During this period oxytocin infusion was done and patient were under antibiotic coverage with vitals monitoring. The cost of conventional balloon tamponade requires Foley's catheter, condom, vicryl or silk for tying which itself costs around 300 rupees. On the other hand, with CG balloon the cost is cut down to Foley's catheter and condom only. For a center with limited resource setting this is very important to consider the monetary benefit. There have been various reports describing the use of balloon tamponade to arrest bleeding from vaginal lacerations, cervical pregnancies and molar pregnancies, inversion. ${ }^{7-10}$ However these cases were managed in wellresourced facilities using proprietary balloon tamponade devices often in combination with other interventions as cerclage placement and methotrexate administration. ${ }^{11}$

\section{CONCLUSION}

Intra uterine balloon tamponade is thus very effective, cheap utility in a low resource setting. With the innovation of $\mathrm{CG}$ balloon the cost factor is also been considered, also the presence of drainage port makes it superior to conventional balloon catheter drill should also include them so as to increase their utilization for the benefit of the women.

\section{Funding: No funding sources}

Conflict of interest: None declared

Ethical approval: Not required

\section{REFERENCES}

1. World Health Organization (WHO). WHO recommendations for the prevention and treatment of postpartum haemorrhage. Geneva: World Health Organization (WHO); 2012.

2. FIGO. FIGO guidelines: prevention and treatment of postpartum hemorrhage in low-resource settings. Int J Gynaecol Obstet. 2012;117:108-18.

3. Georgiou C. A review of current practice in using Balloon Tamponade Technology in the management of postpartum haemorrhage. Hypertens Res Preg. 2014;2:1-10.
4. Mishra N, Agrawal S, Gulabani K, Shrivastava C. Use of an innovative condom balloon tamponade in postpartum haemorrhage: a report. J Obstet Gynecol India. 2016;66(1):63-7.

5. Dalia Y, Agrawal M, Sharma A. Various modifications of condom balloon tamponade and their method, efficacy, outcomes in management of atonic postpartum hemorrhage in tertiary care centrea observational study. JMSCR. 2018;6(5):482-9.

6. Rathore AM, Gupta S, Manaktala U, Gupta S,Dubhey C, Khan AM. Uterine tamponade using condom catheter balloon in the management of Nontraumatic postpartum haemorrhage. J Obstet Gynaecol Res. 2012;38:1162.

7. Tattersall M, Braithwaite W. Balloon tamponade for vaginal lacerations causing severe postpartum haemorrhage. BJOG: Int J Obstet Gynaecol. 2007; 114 (5):647-8.

8. Bakour SH, Thompson PK, Khan KS. Successful conservative management of cervical ectopic pregnancy with combination of methotrexate, mifepristone, surgical evacuation and tamponade using a double balloon three-way catheter. J Obstet Gynaecol. 2005;25(6):616-8.

9. Kolomeyevskaya NV, Tanyi JL, Coleman NM, Beasley AD, Miller HJ, Anderson ML. Balloon tamponade of hemorrhage after uterine curettage for gestational trophoblastic disease. Obstet Gynecol. 2009;113(2):557-60.

10. Yadav S, Malhotra A. Novel use of balloon tamponade saves a patient with uterine inversion in severe shock. Int J Reprod Contracept Obstet Gynecol. 2017;6:5638-41.

11. Makin J, Suarez-Rebling DI, Varma Shivkumar P, Tarimo V, Burke TF. Innovative uses of condom uterine balloon tamponade for postpartum hemorrhage in India and Tanzania. Case Reports Obstet Gynecol. 2018;2018.

Cite this article as: Xess $\mathrm{S}$, Baghel $\mathrm{P}$. An observational study to compare between conventional condom balloon catheter with CG balloon. Int J Reprod Contracept Obstet Gynecol 2019;8:566-9. 